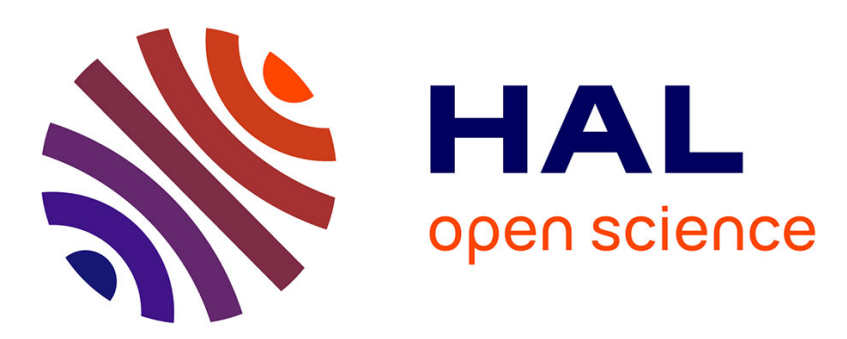

\title{
Détermination des trois paramètres optiques principaux d'un cristal, en grandeur et en direction, par le réfractomètre
}

\author{
A. Cornu
}

\section{- To cite this version:}

A. Cornu. Détermination des trois paramètres optiques principaux d'un cristal, en grandeur et en direction, par le réfractomètre. J. Phys. Theor. Appl., 1902, 1 (1), pp.136-147. 10.1051/jphystap:019020010013600 . jpa-00240584

\section{HAL Id: jpa-00240584 https://hal.science/jpa-00240584}

Submitted on 1 Jan 1902

HAL is a multi-disciplinary open access archive for the deposit and dissemination of scientific research documents, whether they are published or not. The documents may come from teaching and research institutions in France or abroad, or from public or private research centers.
L'archive ouverte pluridisciplinaire HAL, est destinée au dépôt et à la diffusion de documents scientifiques de niveau recherche, publiés ou non, émanant des établissements d'enseignement et de recherche français ou étrangers, des laboratoires publics ou privés. 


\section{DÉTERMINATION DES TROIS PARAMĖTRES OPTIQUES PRINGIPAUX D'UN GRISTAL, EN GRANDEUR ET EN DIREGTION, PAR LE RÉFRACTOMĖTRE (');}

Par II. A. CORNU.

La détermination des trois indices et des trois axes principaux d'un cristal intéresse au plus haut point l'optique, la minéralogie et la pétrographie.

Cette détermination, longue et difficile lorsqu'on opère par la méthode des prismes (qui exige la taille de faces planes rigoureusement orientées), devient théoriquement très simple lorsqu'on opère par réflexion totale dans un milieu suffisamment réfringent, car une seule face plane, orientée d'une manière quelconque, fournit toutes les données nécessaires.

C'est ce qui résulte des travaux de MM. F. Kohlrausch, Quincke, Liebisch, Brill, Ch. Soret; d'autre part, la construction de réfractomètres (Pulfrich, Abbe) a rendu les observations très pratiques.

La mesure des trois indices principaux est donc devenue particulièrement aisée; mais la détermination des trois directions principales est restée jusqu'ici, du moins à ma connaissance $\left({ }^{2}\right)$, hérissée de calculs inabordables pour la pratique courante.

L'éiude géométrique directe de la réflexion totale sur une surface cristalline m'a conduit, pour cette importante application du réfractomètre, à des relations analytiques d'une simplicité inespérée.

Cette étude est fondée sur la construction de la surface de l'onde (indiquée par Fresnel, développée par Ampère et Plücker), qui permet d'obtenir un point et la normale en ce point en partant de l'ellipsoïde dont les axes $a, b, c$, sont les inverses des indices principaux $n_{\lambda}, n_{, j}, n_{z}$ :

(1) $\frac{x^{2}}{a^{2}}+\frac{y^{2}}{b^{2}}+\frac{z^{2}}{c^{2}}=1$ avec $\quad n_{x}=\frac{1}{a}<n_{y}=\frac{1}{b}<n_{z}=\frac{1}{c}$.

La surface de l'onde est le lieu des points obtenus en portant, à partir de l'origine, sur la normale à chaque plan diamétral, la longueur des deux axes de la section elliptique déterminée par ce plan dans l'ellipsoïde.

(1) Communication faite à la Société française de physique : Séance du 6 décembre 1901.

(2) M. Latrexin, Bulletin de la Sociélé francaise de Minéralogie, t. XIV, p. 100 ; 1891. 
Cette construction revient à faire tourner d'un angle droit chaque axe de la section elliptique autour de l'autre axe. Si, dans cette rotation, on entraîne aussi la normale à l'ellipsoïde au sommet correspondant de l'ellipse, cette normale reste dans le plan de rotation et coïncide avec la normale à la surface d'onde au point ainsi déterminé. On construit donc ainsi, outre le point de la surface, la normale en ce point, c'est-à-dire la normale à l'onde plane tangente en ce point à la surface de l'onde.

Conditions relatives $\dot{a}$ la réflexion totale. - Pour qu'une onde plane venant d'un milieu extérieur isotrope pénètre à travers une face plane dans l'intérieur d'un cristal, il faut, d'après la construction d'Huygens :

$1^{\circ}$ Que la normale à l'onde plane réfractée soit dans le plan d'incidence ;

$2^{\circ}$ Que l'on puisse mener, par la trace de l'onde incidente sur la face cristalline, un plan tangent à la surface d'onde du cristal; il faut donc que cette trace ne coupe pas la section de la surface d'onde par la face cristalline : la limite de tangence correspond à la réflexion totale.

Donc, pour déterminer les angles et plans d'incidence de réflexion totale sur une face plane cristalline, il suffit de connaître la section de la surface de l'onde par cette face.

Chaque tangente à cette courbe est la normale d'un plan d’incidence et l'angle de réflexion totalc correspondant I est défini par la distance $p$ à l'origine de cette tangente, suivant la formule :

$$
p \sin \mathrm{I}=\mathrm{R},
$$

$\mathrm{R}$ étant l'inverse de l'indice $\mathrm{N}$ du milieu extérieur.

On peut ainsi mesurer, dans une série d'azimuts $\omega_{1}, \omega_{2}, \ldots$, autour de la normale $\mathrm{O} \approx \mathrm{c}$ à la face cristalline, les distances $p_{1}, p_{2}, \ldots$, et, par suite, déterminer expérimentalement une courbe $p=f^{*}(\omega)$, qui est évidemment la podaire de la section de la surface d'onde.

La connaissance de cette podaire définit complètement cette surface, car les coefficients de son équation (qui est du huitième degré) sont des fonctions connues des trois paramètres $n_{x}, n_{y}, n_{z}$ et des angles $\alpha, \beta, \gamma$ que la normale à la face cristalline fait avec les axes principaux du système; mais la complexité de ces fonctions en rend l'utilisation presque inabordable.

Maxima et minima des angles d'incidence. - L'équation de la 
podaire devient inutile si l'on se borne à l'observation des maxima et minima des angles de réflexion totale et des azimuts correspondants du plan d'incidence, c'est-à-dire des distances $p$ maxima et minima en grandeur et en direction; la construction des rayons vecteurs de la surface de l'onde permet d'en obtenir directement la valeur.

Il suffit de rappeler la génération de la section plane considérée de la surface d'onde: comme tous ses rayons vecteurs sont dans un même plan, les ellipses génératrices sont des sections de l'ellipsoïde par un plan tournant autour de la normale $O$ əo à la face cristalline. On aperçoit alors immédiatement trois valeurs présentant un maximum ou un minimum : en effet, aucune ellipse ne peut avoir d'axe plus grand que $a$ ni plus petit que $c$; par conséquent, l'ellipse

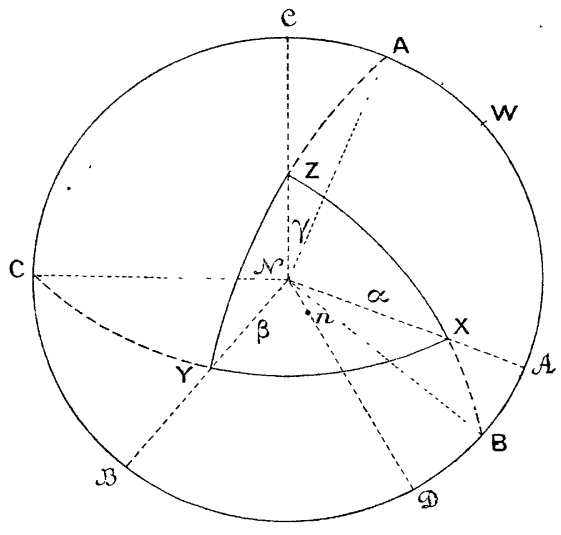

FIG. 1

dont le plan $ぇ$ X ment pour grand axe l'axe maximum $a$, tourné d'un angle droit autour de son petit axe. Il constituera le rayon vecteur maximum

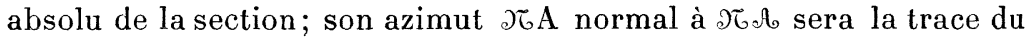
plan d'incidence dont l'angle limite $\mathrm{I}_{x}$ fournit l'indice minimum $n_{x}=\frac{1}{a}$. Il en sera de même pour l'azimut əઢC (à angle droit de la

(1) La figure représente une projection stéréographique sur le plan $\mathrm{ABC}$ de la face cristalline (dont la normale $0 . \tilde{\tau}$ se projette en $\mathfrak{\tau}$ ) des trois plans principaux XY, YZ, ZY de la surface de l'onde. 
projection $\approx € €$ de l'axe $z)$, où l'on observera l'angle limite $\mathrm{I}_{z}$ correspondant à l'indice maximum $n_{z}=\frac{1}{c}$.

Par raison de symétrie, l'axe moyen $b$ sera aussi l'un des axes d'une section elliptique : il fournira dans l'azimut )厄B (à angle droit de $\tau_{6}(l)$, projection de l'axe $y$ ) un angle limite $\mathrm{I}_{y}$ correspondant à l'indice moyen $n_{y}=\frac{1}{b}$. Mais cet angle sera, suivant les cas, un maximum ou un minimum.

En résumé, les trois indices principaux $n_{x}, n_{y}, n_{z}$ sont donnés par les incidences $\mathrm{I}_{x}, \mathrm{I}_{y}, \mathrm{I}_{z}$ respectivement observées dans les plans $\widetilde{\tau} \mathrm{A}$, $\jmath \mathrm{B}, \jmath \mathrm{C}$, traces sur la face cristalline des plans principaux : $\tau \mathrm{A}$ trace de YZ, эぇB de ZX, эぇC de XY.

Mais la section plane de la surface d'onde, étant une courbe centrée du quatrième degré, doit présenter quatre rayons vecteurs alternativement maximum ou minimum en valeur absolue. On doit done trouver un quatrième plan d'incidence fournissant un angle limite I maximum ou minimum correspondant à un indice intermédiaire v.

Or, parmi tous les plans diamétraux passant par la normale $\mathrm{O} \cdot \tilde{\mathcal{G}}$, il en est un qui se distingue de tous les autres : c'est le plan $) \lessdot(\mapsto$, qui contient la normale à l'ellipsoïde au point où $O$, 厄 coupe la surface : c'est le quatrième plan d'incidence cherché; car il remplit toutes les conditions requises.

En effet, sa trace $\succsim \curvearrowleft($ est la normale d'une section elliptique dont un des sommets est en $\approx \tau$, puisque la normale à l'ellipsoïde en $\tau$ se projette sur cette section suivant $O \Im$ : donc $O \approx$ est un axe de l'ellipse. La rotation autour de l'axe, s'effectuant dans le plan $\succsim \circlearrowleft$, entraine dans ce plan la normale à l'ellipsoïde, qui devient la normale à la surface d'onde au point de la section plane que cette construction détermine. La normale en ce point se projette donc sur la section plane suivant la direction $\Im \widetilde{G}(\odot$; donc le rayon vecteur ayant pour longueur $\mathrm{O} \approx$ (diamètre de l'ellipsoïde normal à la surface cristalline) est maximum ou minimum : C. Q. F. D.

Le quatrième plan d'incidence $\widetilde{\sigma}(\partial$ s'obtiendra donc sur la projection stéréographique en joignant par un grand cercle $\varkappa$ au point $n$ représentatif de la direction de la normale àl'ellipsoïde au point $\tau_{6}$ et prolongeant jusqu'en $\odot$, plan de la surface cristalline.

Les observations au réfractomètre fournissent les azimuts $\mathrm{A}, \mathrm{B}$, $\mathrm{C}, \circledast$ et, dans chacun d'eux, les angles limites $\mathrm{I}_{x}, \mathrm{I}_{y}, \mathrm{I}_{z}, \mathrm{I}$. 
Calcul des trois indices el vérification. - Le calcul des indices n'exige que la détermination des angles de réflexion totale. Si l'on désigne par $\mathrm{N}$ l'indice de réfraction du milieu extérieur, on a :

(2) $n_{x}=\mathrm{N} \sin \mathrm{I}_{x}, \quad n_{y}=\mathrm{N} \sin \mathrm{I}_{y}, \quad n_{z}=\mathrm{N} \sin \mathrm{I}_{z}, \quad \nu=\mathrm{N} \sin \mathrm{I}$,

Il se présente une ambiguïté entre $n_{y}$ et $\nu$, intermédiaires entre $n_{x}$ et $n_{\xi}$. On la lève aisément si l'on connaît les angles $\alpha, \beta, \gamma$ que fait la normale $\pi$ à la face cristalline avec les trois axes $x, y, z$.

En effet, le rayon vecteur $r$ de l'ellipsoïde (1), suivant la direction $\mathrm{O}, \tilde{T}$, est précisément celui de la section de la surface d'onde dans l'azimut $\mathscr{\jmath} \mathscr{O}$; l'inverse de ce rayon vecteur est égal àl'indice calculév.

Or on a, pour le point $\mathfrak{d}$,

d'où :

$$
x=r \cos \alpha, \quad y=r \cos \beta, \quad z=r \cos \gamma,
$$

ou

$$
\left(\frac{\cos ^{2} \alpha}{a^{2}}+\frac{\cos ^{2} \beta}{b^{2}}+\frac{\cos ^{2} \gamma}{c^{2}}\right)=\frac{1}{r^{2}} .
$$

$$
\nu^{2}=n_{x}^{2} \cos ^{2} \alpha+n_{\breve{y}}^{2} \cos ^{2} \beta+n_{\tilde{\nu}}^{2} \cos ^{2} \gamma,
$$

relation qui permet de calculer $\vee^{2}$, de lever l'ambiguïté (1) et d'obtenir une précieuse vérification.

Calcul des trois directions principales. - Si l'on ne connaît pas les trois directions principales, e'est-à-dire l'orientation des axes $\mathrm{OX}, \mathrm{OY}, \mathrm{OZ}$, on pourra lẹs déduire de l'observation des azimuts $\mathrm{A}$, $\mathrm{B}, \mathrm{C}, \oplus$, rapportés dans le plan de la face cristalline à une orientation cristallographique quelconque $\pi \mathrm{W}$.

Les formules suivantes résolvent le problème de plusieurs manières.

Connaissant les azimuts A, B, C, on en déduit les angles $\alpha, \beta, \gamma$ que fait la normale à la face $\tau^{6}$ avec les trois axes $\mathrm{OX}, \mathrm{OY}, \mathrm{OZ}^{\prime}$ :

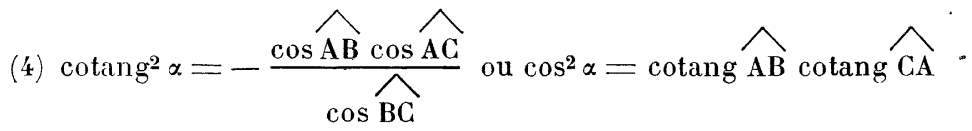

et les deux autres par permutation tournante.

(i) Pratiquement, on la lève en répétant les observations sur une autre face cristalline : sur les quatre indices calculés, trois reproduisent les valeurs déjả obtenues $n_{x}, n_{y}, n_{z}$; les deux non identifiables sont $\nu$ et $\nu^{\prime}$ qui dépendent de $\alpha \beta \gamma$ ou $\alpha^{\prime} \beta^{\prime} \gamma^{\prime}$. 
On vérifie le calcul en remarquant que :

$$
\cos ^{2} \alpha+\cos ^{2} \beta+\cos ^{2} \gamma=1 \text {. }
$$

L'orientation cristallographique des trois axes est alors complètement définie, puisqu'on suppose connue l'origine des azimuts $\mathrm{A}, \mathrm{B}$, $\mathrm{C}$ par rapport à une direction de repère $\tau \mathrm{W}$.

Il importe également de déterminer l'azimut $)(\mathcal{C}$ du plan d'incidence du quatrième indice : on obtient la valeur des angles $\widehat{A}(\Omega$,

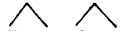

$\mathrm{B} \oplus, \mathrm{C} \Theta$, par des expressions de la forme :

$$
\operatorname{tang} \widehat{\mathrm{A} \Theta}=\frac{\cos \alpha}{\cos \beta \cos \gamma} \frac{n_{x}^{2}-\nu^{2}}{n_{y}^{2}-n_{\tilde{z}}^{2}}=\operatorname{tang} \widehat{\mathrm{BC}} \frac{n_{x}^{2}-\nu^{2}}{n_{y}^{2}-n_{\tilde{\nu}}^{2}} .
$$

Ces formules tombent en défaut quand la normale $) \tau$ à la face utilisée coöncide avec l'un des plans principaux. L'un des facteurs $\cos \alpha$, $\cos \beta, \cos \gamma$ devient nul, ce dont on est prévenu par la rectangularité ou l'égalité de certains azimuts A, B, C, $\mathcal{~}$. Mais alors le problème est beaucoup plus simple : l'expression (3) du quatrième indice suffit alors pour déterminer la position de la normale $) \tau$ dans le plan de ces axes.

Je me suis assuré, par de nombreuses observations au réfractomètre Abbe sur divers cristaux, que ces formules sont exactes à lordre d'approximation corrélative de celle des données expérimentales. J'ai opéré le plus souvent sur de beaux cristaux clinorhombiques d'acide tartrique du commerce, qui présentent une birélringence considérable, condition nécessaire pour unevérification efficace des formules.

\section{DÉMONSTRATION ET USAGE DES FORMULES.}

Les formules données précédemment (p. 140-141) pour la détermination complète en grandeur et en direction des paramètres optiques d'un cristal peuvent ètre établies d'une manière assez simple.

Le premier groupe (4) est purement géométrique: il fournit les angles $\alpha, \beta, \gamma$, que la normale $\approx \sigma$ à la face cristalline $(f i g .1)$ fait avec les trois axes principaux $\mathrm{X}, \mathrm{Y}, \mathrm{Z}$, indépendamment de la grandeur des paramètres $\left(a=\frac{1}{n_{x}}, b=\frac{1}{n_{y}}, c=\frac{1}{n_{z}}\right)$ de l'ellipsoïde qui caractérise la surface de l'onde lumineuse dans le cristal. 
Le second groupe (ä), au contraire, dépend essentiellement de ces paramètres en grandeur et en direction, ainsi que la formule (3) établie au cours de l'analyse du phénomène.

Premier groupe. - La projection stéréographique ci-après fournit immédiatement les triangles sphériques qui lient les angles inconnus $\alpha, \beta, \gamma$ aux données de l'observation; ces données sont les azimuts $\pi \mathrm{A}, \tau \mathrm{B}, \tau \mathrm{C}$ des trois plans de réflexion dans lesquels on a mesuré les incidences qui ont fourni respectivement les indices $n_{x}$, $n_{y}, n_{z}$.

La normale $)_{0}$ à la face réfléchissante (supposée dans l'intérieur du trièdre trirectangle des axes, comme sur la figure, hypothèse que. l'on peut toujours faire) est le sommet de trois triangles rectila-

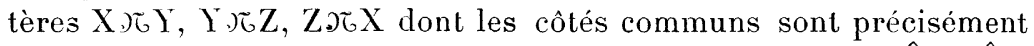

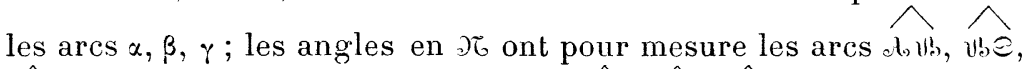

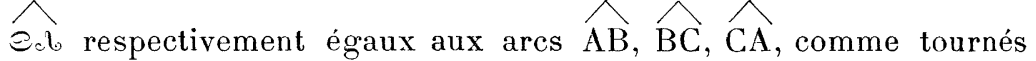
dans lè même sens de $90^{\circ}$ (Il n'en scrait pas ainsi dans le cas où $\mathfrak{r}$ serait en dehors du triangle XYZ). Ainsi dans $\mathrm{X} \curvearrowright \tau B$, on a la relation :

$$
0=\cos \alpha \cos \beta+\sin \alpha \sin \beta \cos \widehat{A B},
$$

d'où l'on tire $\cos \widehat{\mathrm{AB}}$ et les deux autres cosinus analogues, par permutation tournante, car la permutation s'applique dans le même sens à l'ordre de succession des points $\mathrm{A}, \mathrm{B}, \mathrm{C}: \mathrm{XYC}, \mathrm{YZA}, \mathrm{ZXB}$. Il vient donc :

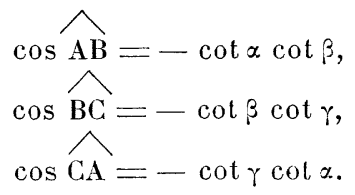

Divisons la seconde équation par le produit des deux autres; il vient:

$$
\operatorname{tang}^{2} \alpha=-\frac{\widehat{\cos \widehat{B C}}}{\widehat{\cos \widehat{A B} \cos \widehat{C A}}} \text {. }
$$

C'est la première formule du groupe (4) (p.140):

Les trois angles $\widehat{\mathrm{AB}}, \widehat{\mathrm{BC}}, \widehat{\mathrm{CA}}$ ayant entre eux la relation:

$$
\widehat{\mathrm{AB}}+\widehat{\mathrm{BC}}+\widehat{\mathrm{CA}}=360^{\circ}
$$


on peut éliminer l'un d'eux dans la formule $\left(\mathbf{4}_{a}\right)$, par exemple $\widehat{\mathrm{BC}}$,

$$
\cos \widehat{\mathrm{BC}}=\cos (\widehat{\mathrm{AB}}+\widehat{\mathrm{CA}})
$$

Substituant et réduisant, il vient (? 1$)$ :

$$
\left\{\begin{array}{l}
\cos ^{2} \alpha=\cot \widehat{\mathrm{AB}} \cot \widehat{\mathrm{CA}}, \\
\cos ^{2} \beta=\cot \widehat{\mathrm{BC}} \cot \widehat{\mathrm{AB}}, \\
\cos ^{2} \gamma=\cot \widehat{\mathrm{CA}} \cot \widehat{\mathrm{BC}},
\end{array}\right.
$$

valeurs qui satisfont d'elles-mêmes à la condition :

$$
\cos ^{2} \alpha+\cos ^{2} \beta+\cos ^{2} \gamma=1 \text {. }
$$

Ces formules, purement géométriques, sont théoriquement à l'abri de toute difficulté dans leur emploi; mais, en pratique, l'incertitude inévitable que présente la détermination de l'azimut d'un maximum ou d'un minimum arnène parfois des embarras dont il faut être prévenu.

Ainsi il peut arriver que les données de l'observation substituées dans les formules $\left(\boldsymbol{4}_{i}\right)$ conduisent à des valeurs négatives pour les carrés des cosinus, c'est-à-dire à des solutions imaginaires pour les angles $\alpha, \beta$ ou $\gamma$. Or, d'après la nature du problème, ces valeurs sont nécessairement réelles $\left({ }^{2}\right)$; il faut donc que les trois cotangentes soient de même signe; par suite, les angles $\widehat{\mathrm{AB}}, \widehat{\mathrm{BC}}, \widehat{\mathrm{CA}}$, ramenés entre $0^{\circ}$ et $90^{\circ}$ par addition ou soustraction de $180^{\circ}$ doivent aussi

(1) On peut, par une simple construction géométrique, obtenir ces angles $x, \beta$, $\gamma$, c'est-à-dire déduire directement les points $\mathrm{X}, \mathrm{Y}, \mathrm{Z}$ des points $\mathrm{A}, \mathrm{B}, \mathrm{C}$, en superposant à la projection stéréographique une projection orthogonale auxiliaire sur le plan de la face cristalline, qui est ici le plan de la figure. On sait, en effet, que la projection orthogonale du sommet d'un trièdre trirectangle sur un plan qui coupe ses trois arêtes est le point de concours des trois hauteurs du triangle. intersection des trois faces avec le plan sécant. On reconnait aisément que les trois côtés de ce triangle sont paralièles aux directions $\left.) \widetilde{G},)^{6},\right)_{G} \mathrm{G}$ et les

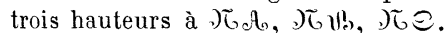

Prenant le point $\pi$ conme point de concours des trois hauteurs $) \tau d, \pi$, $)$,

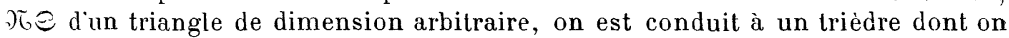
construit tous les éléments angulaires par des rabattements évidents.

() La construction géométrique précitée en donne la preuve : la construction du triangle auxiliaire dont les directions des trois hauteurs sont données est toujours possible. 
ètre de même signe. Cette condition fournit un contrôle qualitatif de l'exactitude des observations; si elle n'est pas remplie, c'est qu'il s'est glissé quelque erreur; il y a lieu alors de reviser les observations et, au besoin, de recommencer les mesures jusqu'à ce que les azimuts satisfassent à la condition de réalité du cosinus.

Il y a cependant un cas où l'on n'y parvient pas d'une manière satisfaisante, à cause de l'incertitude des observations. C'est celu où certaines différences d'azimut sont très voisines de $90^{\circ}$; alors une petite erreur dans les mesures peut rendre un angle plus grand ou plus petit que $90^{\circ}$ et, par conséquent, faire passer la cotangente du signe positif au négatif. Mais on est averti par là du fait que la face cristalline utilisée est presque parallèle à l'un des ax́es principaux : les valeurs des produits des cotangentes sont indéterminées; dès lors cette face, bien que susceptible de fournir les trois indices principaux, est, par son orientation, impropre à la détermination précise des trois directions principales, du moins à l'aide des formules $\left(4_{b}\right)$.

On est alors amené à supposer que, au moins comme première approximation, l'un des angles $\alpha, \beta, \gamma$, par exemple $\alpha$, est égal à $90^{\circ}$ exactement; et, par suite, $\cot \alpha=0$.

Il reste la relation:

$$
\cos ^{2} \beta^{\prime}+\cos ^{2} \gamma^{\prime}=1 \quad \text { ou } \quad \beta^{\prime}+\gamma^{\prime}=90^{\circ} .
$$

On utilise alors la valeur du quatrième indice $ə$ pour la détermination de $\beta^{\prime}$, laquelle demeure la seule inconnue.

L'équation :

$$
\nu^{2}=n^{2} x \cos ^{2} \alpha+n^{2} y \cos ^{2} \beta+n^{2} z \cos ^{2} \beta,
$$

peut en effet se mettre sous la forme

$$
\nu^{2}\left(\cos ^{2} \beta^{\prime}+\sin ^{2} \xi^{\prime}\right)=n_{y} \cos ^{2} \beta^{\prime}+n_{2}^{2} \sin ^{2} \beta^{\prime} \text {. }
$$

d'où l'on tire:

$$
\tan g^{2} \beta^{\prime}=\frac{\nu^{2}-n^{2} z}{n^{2} y-\nu^{2}}
$$

expression (') qui achève de déterminer l'orientation de la normale à la face cristalline dans l'hypothèse où cette face est rigoureusement parallèle à l'axe OY $\left(\alpha=90^{\circ}, \beta=\beta^{\prime}, \gamma^{\prime}=90^{\circ}-\beta^{\prime}\right)$.

En général, l'hypothèse $\alpha=90^{\circ}$ ne sera qu'approchée et, bien que

(1) Étant homogène par rapport à $n_{y}, n_{z}$ et $\nu$, la valeur de $\beta^{\prime}$ est indépendante de la valeur de l'indice de réfraction de la substance réfringente auxiliaire des réfractomètres. 
la valeur de $f^{\prime}$ soit plus exacte qu'on ne pourrait le penser, la conclusion qu'on tirera de ces mesures sera la nécessité de recourir à une autre face cristalline orientée dans une direction plus favorable, afin d'obtenir des données meilleures et une vérification des résultats obtenus. Lorsqu'on est maître de choisir cette orientation, on prend, pour $\mathrm{O}) \tau$, la direction qui lait, avec les axes OX, OY, O\% (supposés connus approximativement), des angles sensiblement égaux.

Remarques. - En dehors du cas particulier qui vient d'être signalé, on ne rencontre pas, en général, d'autre difficulté que l'ambiguïté existant entre l'indice moyen $n_{y}$ et le quatrième indice $v$.

Dans ce qui précède, on l'a supposée levée par des considérations étrangères aux mesures; mais cette ambiguïté n'entraîne d'autre inconvénient que celui d’un calcul supplémentaire. En effet, si l'on n'a aucun motif de distinguer $n_{y}$ de $v$, on considère arbitrairement l'un des deux indices comme $n_{l j}$ et, à l'aide des trois azimuts correspondant aux trois indices $n_{x}, n_{y}, n_{z}$ adoptés, on calcule les angles $\alpha$, $\beta, \gamma$. Si le choix est bon, l'équation (3) vérifiera la valeur du quatrième indice, considéré comme représentant $\nu$; sinon, on intervertira $n_{\dot{y}}$ et $\nu, \mathrm{B}$ et (id), et l'on recommencera le calcul de $\alpha, \beta, \gamma$; la vérification de (3) devra alors être satisfaisante.

Second groupe. - Le second groupe de formules (̋̈) est destiné à fournir des vérifications en utilisant les données relatives au quatrieme indice dont on mesure, d'une part, la grandeur $v$, d'autre part lazimut $\Im \tau\left(\theta_{c}\right.$ du plan de réflexion où on l'observe.

L'équation (3), déjà employée, doit être rangée dans ce groupe, bien qu'elle ait été établie directement, car elle constitue aussi une relation de contrôle entre les indices $n_{x}, n_{y}, n_{z}$, v et les angles $\alpha, \beta, \gamma$.

Celles qu'il reste à démontrer se rapportent aux azimuts $\approx \Lambda, \approx B$, $\approx$ C et $) \widetilde{\tau}(\partial$; la définition géométrique de la surface de l'onde déduite de l'ellipsoïde établit entre eux des relations qui permettent de cal-

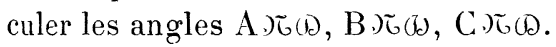

La démonstration de ces formules se réduit à calculer les cosinus directeurs des diverses directions nécessaires pour obtenir l'expression des angles qu'elles font entre elles. Ce calcul un peu laborieux n'offre, d'ailleurs, aucune difficulté.

Direclion ) mut $\tilde{G}($ est la trace du plan qui passe par la normale $n$ à l'ellipsoïde, au point où la normale oć à la face réfléchissante rencontre cette surface.

J. de Phys., $4^{\circ}$ série, t. I. (Mars 1902.) 
Calculons d'abord les cosinus directeurs $\xi^{\prime}, \eta^{\prime}, \zeta^{\prime}$ de la formule $\Re \Theta^{\prime}$ à ce plan (non tracée sur la figure) rapporté aux axes X, Y, Z.

Ils sont déterminés par les trois conditions:

$$
\xi^{\prime 2}+\eta^{\prime 2}+\zeta^{\prime 2}=1, \quad \xi^{\prime} \alpha+\eta^{\prime} \beta+\zeta^{\prime} \gamma=0, \quad \xi^{\prime} \frac{\alpha}{a^{2}}+\eta^{\prime} \frac{\beta}{b^{2}}+\zeta^{\prime} \frac{\gamma}{c^{2}}=0
$$

qui expriment, outre la rectangularité des axes, que )ढ( $\delta^{\prime}$ est normale à ) $)$ et à $n$ (on a remplacé, pour abréger l'écriture, $\cos \alpha, \ldots$, par $\alpha, \beta, \gamma)$; d'où l'on tire:

$$
\frac{\xi^{\prime}}{\beta \gamma\left(n_{z}^{2}-n_{y}^{2}\right)}=\frac{r_{i}^{\prime}}{\gamma \alpha\left(n_{x}^{2}-n_{z}^{2}\right)}=\frac{\zeta^{\prime}}{\alpha_{i}^{\beta}\left(n_{y}^{2}-n_{x}^{2}\right)}=1
$$

expressions où les carrés des indices principaux remplacent les inverses des carrés des axes de l'ellipsoïle.

D'après les relations bien connues entre les neuf cosinus d'un trièdre trirectangle, on écrira immédiatement les cosinus directeurs $\xi, \eta, \zeta$ de la trace $)(B$ du plan $) \tau n$ sur le plan de la face cristalline, car elle forme un trièdre trirectangle avec $\tilde{\tau}(\alpha, \beta, \gamma)$ et (B) $\left(\xi^{\prime}, \eta^{\prime}, \zeta^{\prime}\right)$ :

$$
\xi=\xi \zeta^{\prime}-\gamma \gamma_{i}^{\prime}, \quad r_{1}=\gamma \xi^{\prime}-\alpha \zeta^{\prime}, \quad \zeta=\alpha \gamma_{1}^{\prime}-\beta \xi^{\prime} .
$$

Iprès substitution et rédıction en ayant égard à l'équation (3), on trouve:

$$
\frac{\xi}{\alpha\left(\nu^{2}-n_{x}^{2}\right)}=\frac{r_{i}}{3\left(\nu^{2}-n_{y}^{2}\right)}=\frac{\zeta}{\gamma^{2}\left(\nu^{2}-r_{1}^{2}\right)}=\frac{1}{R},
$$

le radical $\Re$ étant évidemment le même que pour $\left(^{\prime}\left(\zeta^{\prime}, \eta^{\prime}, \zeta^{\prime}\right)\right.$.

Directions $\approx \mathrm{A}, \tau_{\mathrm{B}}, \tau_{\mathrm{C}}$ - - Calculons, par exemple, les cosinus directeurs de $\tau \mathbf{A}\left(\mathrm{X}_{u}, \mathrm{Y}_{a}, \mathrm{Z}_{u}\right)$, les autres s'obtiendront par permutation tournante. $\approx .1$ est la normale au plan qui passe par $\approx(\alpha, \beta, \gamma)$ et par l'axe OX $(\mathbf{1}, \mathbf{0 , 0})$ : d'où les trois conditions :

$$
\mathrm{X}_{a}^{2}+\mathrm{Y}_{a}^{2}+\mathrm{Z}_{a}^{2}=1, \quad \mathrm{X}_{a} \alpha+\mathrm{Y}_{a} \beta+\mathrm{Z} \alpha \gamma=0, \quad \mathrm{X}_{a}=0,
$$

d'où :

$$
\frac{X_{a}}{0}=\frac{Y_{a}}{\gamma}=\frac{Z_{a}}{-\beta}-\frac{1}{\sqrt{\gamma^{2}-\gamma^{2}}}
$$

On en conclut:

$$
\mathrm{X}_{a}=0, \quad \mathrm{Y}_{a}=\frac{\gamma}{\sqrt{1^{2}-\gamma^{2}}}, \quad \mathrm{Z}_{a}=\frac{-\beta}{\sqrt{\sigma^{2}-\gamma^{2}}} .
$$


Calculs des angles $\widehat{\mathrm{A}(\mathrm{\theta})}, \widehat{\mathrm{B}(\hat{\mathrm{C}})}, \widehat{\mathrm{C}(\mathrm{l})}$. - On a évidenment, à l'aide des valeurs calculées ci-dessus :

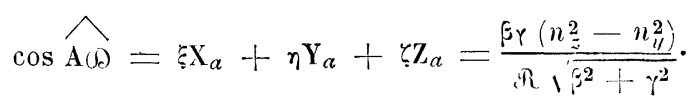

Le radical $\mathbb{R}_{\text {est }}$ compliqué et se prète mal au calcul numérique. On · tourne cette difficulté en utilisant $\cos \mathrm{A}\left(\partial^{\prime}\right.$, qui est égal et de signe contraire à $\sin A(\hat{A}$, puisque $) \tau(B)$ et $) \tau\left(\bar{d}_{c}\right)^{\prime}$ sont rectangulaires et que $\widehat{\mathrm{A} O}$ et $\widehat{\mathrm{A} \circlearrowleft \partial^{\prime}}$ sont comptés dans le mème sens que $\widehat{\mathrm{AB}}, \widehat{\mathrm{BC}}, \widehat{\mathrm{CA}}$,

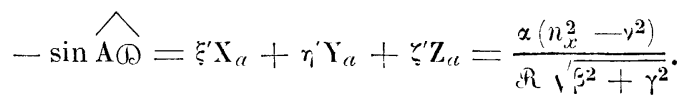

Divisant membre à membre, il vient, après aroir remis $\cos \alpha, \cos \beta$, $\cos \%$ au lieu de $\alpha, \beta, \gamma$,

$$
-\operatorname{tang} \widehat{A}=\frac{\cos x}{\cos \beta \cos \gamma} \frac{n_{x}^{2}-y^{2}}{n_{z}^{2}-n_{y}^{2}},
$$

et les deux autres expressions analogues de $\widehat{B}(1), C(B)$.

On les simplifie encore en remplaçant les cosinus par leur valeur tirée de $\left(4_{b}\right)$ :

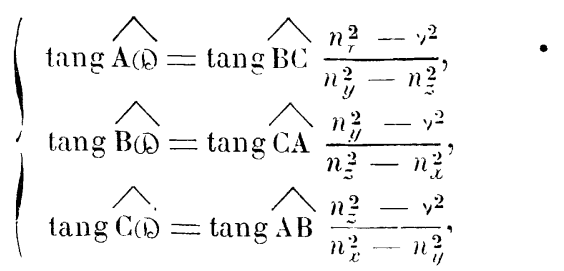

expressions très simples, qui fournissent un contrôle précieux de l'ensemble des paramètres optiques du cristal, car les trois angles calculés ci-dessus ont été directement observés; la valeur de y est celle qui est calculée par l'équátion $(3)$.

Pour achever la détermination de la constitution optique du cristal, il reste à définir l'orientation des trois axes $\mathrm{X}, \mathrm{Y}, \mathrm{Z}$, par rapport aux axes cristallographiques. Or la direction de repère $\pi \mathrm{W}$, observée en

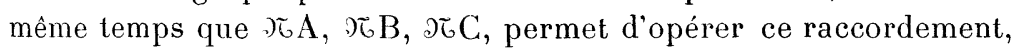
car l'orientation cristallographique de la face .t est supposée connue ainsi que $\approx \mathrm{W}$. Il ne reste plus alors à effectuer que des calculs familiers aux cristallographes. 